\title{
State-of-the-Art Therapy for Glioblastoma Multiforme
}

\author{
A report by \\ Henry S. Friedman, MD, James B. Powell, Jr. Professor of Neuro-Oncology \\ David A. Reardon, MD, Associate Professor of Pediatrics and Surgery
}

$2 / 2 / 2009$

The treatment of patients with glioblastoma multiforme (GBM) is conventionally considered to be a palliative venture with no hope of cure. Traditionally, patients are treated with maximal surgical resection based on the premise that, although surgery is not a curative procedure, a major resection provides for a longer survival and better quality of life. ${ }^{1}$ Radiotherapy increases the duration of survival, but again is not a curative intervention. ${ }^{2}$ The role of chemotherapy, specifically focusing on a foundation of chloroethylating agents such as carmustine (BCNU) or lomustine (CCNU), has been controversial with an equal number of clinicians arguing in favor of or against this treatment. Meta-analysis makes it clear that there is a small increase in median survival associated with the addition of these agents, but a consensus was never reached regarding their use. ${ }^{3}$

Unfortunately, the underlying assumption of virtually all clinicians that GBM is not a curable tumor led to a wide spectrum of interventions utilized in the community to treat these patients. Many patients received a biopsy rather than a major resection of their tumor. Despite the lack of evidence supporting whole-brain versus focal radiotherapy, some patients received (or still receive) whole-brain radiotherapy with the associated increased morbidity. Finally, community and academic physicians chose to not use chemotherapy without a firm foundation of data to support withholding it.

A positive step forward started with the synthesis and evaluation of temozolomide and subsequent phase I and II trials with this methylating agent in the UK. Temozolomide was shown to have activity in phase I trials in two patient populations: those with malignant glioma or those with melanoma. ${ }^{4}$ Subsequent phase II trials in the UK confirmed this activity, ${ }^{5}$ leading Schering Plough to license the drug for studies in the US. Following phase I trials in adults and children, a pivotal registration trial in patients with GBM, and also anaplastic astrocytoma in a separate trialas well as a trial of newly diagnosed patients with GBM with residual disease following initial surgical intervention or biopsy intervention-were initiated. The first published study by Friedman et al. ${ }^{6}$ demonstrated the profound activity of temozolomide in patients with newly diagnosed GBM with a response rate exceeding 50\%. Although the registration trial for patients with firstrelapse GBM demonstrated a near tripling of the six-month progression-free survival, concerns voiced by the US Food and Drug Administration (FDA) regarding this as a legitimate end-point prevented the approval of temozolomide for this indication. ${ }^{7}$ However, the one-arm study evaluating temozolomide in the treatment of patients with first-relapse anaplastic astrocytoma led to an accelerated approval for this agent. ${ }^{8}$

Roger Stupp took the next step forward by building on the results of the prior studies of temozolomide. A one-arm trial was initiated of 65 patients who received surgery then radiation with daily temozolomide, ${ }^{9}$ followed by six cycles of traditional five-days-a-month temozolomide' These results showed a provocative survival curve leading to a randomized phase III trial in patients with newly diagnosed GBM. The results confirmed the benefit of temozolomide, which produced a modest increase in survival for patients receiving this agent. ${ }^{10}$ Overnight, temozolomide became the global standard of care. Ironically, an agent producing a very similar increase in survival-notably Gliadel wafers, which release BCNU into a tumor cavity following their implantation has not been as universally accepted. ${ }^{11}$ Although this may have initially 
reflected concerns with toxicity-due to a lack of appreciation of the need for a watertight dural seal following placement of these wafers and the initially unfavorable cost, at least in the US, it is now clear that both of these problems have been addressed and the use of this agent appears to be increasing.

The problem that we currently face in the field is the continued belief that patients with GBM will die, and therefore our efforts are merely palliative. Accordingly, patients are treated routinely in the community with surgery followed by radiation and temozolomide, followed by temozolomide with only a small hope of long-term survival. It is absolutely ludicrous to believe that any malignancy, particularly one as aggressive as GBM, can be cured with single-agent chemotherapy. Nevertheless, this standard of care persists because there is a concern that there are no other active agents, and the belief that only patients on clinical trials should receive agents that are not approved by the FDA to counter a specific malignancy. Although the community standard of care in the US for oncology patients is to utilize commercially available agents in an off-label setting when data support their use, in many academic neurooncology programs there is major resistance to this approach. This problem can be dealt with in two ways. The first is to design clinical trials using multiple agents in the treatment of GBM. The study by La Rocca et al. is a step in the right direction since it evaluates the use of surgery with placement of Gliadel wafers followed by radiation and temozolomide, followed by temozolomide as a single agent in the treatment of patients with newly diagnosed GBM. ${ }^{12}$

The optimal approach that will hopefully lead to advances in the treatment of patients with GBM is the conduct of randomized phase 3 trials comparing the current standard approach to rationally chosen, investigational therapeutic strategies. Two such trials are currently accruing patients and merit prioritization for patient enrollment. First, Neuradiab is an investigational agent that is a monoclonal antibody that locally delivers radiation to tumor cells by binding to an extracellular matrix protein (tenascin) seen in virtually all GBMs. The antibody is conjugated to iodine-131 and instilled into the resection cavity via a Rickham reservoir following a gross total resection to destroy residual tumor cells left after resection. ${ }^{13}$ Second, cilengitide is an intravenously administered investigational agent that inhibits integrins $\alpha_{v} \beta_{3}$ and $\alpha_{v} \beta_{5}$ that are overexpressed by most GBMs and mediate important tumor cell functions including survival, migration and blood vessel formation (angiogenesis). Cilengitide has shown consistent benefit across a series of phase I and II GBM clinical trials and has very little toxicity. ${ }^{13}$ The standard arm in each of these phase 3 trials is surgery, radiotherapy and temozolomide whereas the investigational arm is the same plus the investigational agent. Patients should unequivocally seek enrollment in these trials or others that will certainly be developed in the future. Unfortunately many newly diagnosed patients will not be eligible for a trial or disappointingly, have insurance coverage that will not allow participation in a trial.

Patients not on a clinical trial can also be treated with off-label commercially available agents, particularly if other studies will evaluate these agents in formal clinical trials, so we are not slowing the progress of the field, but offering patients hope who are not on a trial. An example of this would be the use of Bevacizumab, which in recent studies in combination with irinotecan has been shown to have an extraordinary response rate and duration of response. ${ }^{14,15}$ Although phase III trials that evaluate this agent in newly diagnosed patients will be forthcoming, there is no reason why patients who are not protocoleligible cannot receive this as part of their standard of care. We follow this strategy of using off-label commercially available drugs at Duke and, between 2003 and 2006, 85 patients were treated with surgery, Gliadel wafers (if anatomically appropriate), and radiation therapy with concomitant temozolomide, followed by a rotation of temozolomide, CCNU, and irinotecan. These results are promising and prevent the all too frequent occurrence that only patients on clinical trials are offered optimistic therapy. ${ }^{16}$ We now routinely consider Bevacizumab with or without irinotecan in the treatment of all newly 
diagnosed patients with GBM who are not enrolled on clinical trials. Of course, we argue that additional agents will be required to improve overall outcome for all patients. Ongoing and planned phase 3 trials will provide the key answers about which agents will help provide the next steps forward.

An additional concern that has arisen following the universal acceptance of temozolomide has been the role of $\mathrm{O}^{6}$-methylguanine-DNA methyltransferase (MGMT)-also known as $\mathrm{O}^{6}$ alkylguanine DNA alkyltransferase-in making decisions regarding the use of temozolomide. A series of clinical studies have clearly demonstrated the role of this protein in predicting response to temozolomide. Friedman et al. ${ }^{6}$ showed this first in the upfront study of patients with newly diagnosed GBM. Roger Stupp built on this with subsequent trials further defining the relationship between MGMT and temozolomide. Unfortunately, it is not an all or nothing relationship, particularly when using the promoter methylation assay. It is clear that any recommendations to use or not use temozolomide based on this assay are inappropriate. Maxwell et al. ${ }^{17}$ clearly demonstrated that even in a situation where the methylation assay actually showed a lack of methylation of the promoter, there is still a population of cells that do not stain for MGMT and, therefore, are likely to be sensitive to temozolomide. The appropriate recommendation when the MGMT promoter is not methylated is not to withhold temozolomide, but to use it while including additional agents that will not be susceptible to this protein.

Patients with GBM are not universally incurable, with an ever-increasing, albeit small, fraction of patients who appear to survive the disease. The classical wisdom of utilizing multiple therapeutic agents with non-overlapping toxicity and independent mechanisms of action unequivocally is producing an ever-increasing cohort of patients for whom GBM is not a terminal event. The list of highly promising new treatment agents and strategies continues to grow. Ongoing clinical trials offer great hope to define a future of better outcome and ultimately cure for GBM patients.

\section{REFERENCES}

1. Lacroix M, Abi-Said D, Fourney DR, et al. A multivariate analysis of 416 patients with glioblastoma multiforme: prognosis, extent of resection, and survival. J Neurosurg 2001;95:190-98.

2. Fine HA. The basis for current treatment recommendations for malignant gliomas. J Neuroocol 1994;20:111-20.

3. Stewart LA. Chemotherapy in adult high-grade glioma: a systematic review and metaanalysis of individual patient data from 12 randomised trials. Lancet 2002;359:1011-18.

4. Newlands ES, Blackledge GR, Slack JA, et al. Phase I trial of temozolomide (CCRG 81045: M\&B 39831: NSC 362856). Br J Cancer 1992;65:287-91.

5. O'Reilly SM, Newlands ES, Glaser MG, et al., Temozolomide: a new oral cytotoxic chemotherapeutic agent with promising activity against primary brain tumours. Eur J Cancer 1993;29A:940-42 
6. Friedman HS, Mclendon RE, Kerby T, et aI. DNA mismatch repair and 06alkylguanine.DNA alkyltransferase analysis and response to Temodal in newly diagnosed malignant glioma. J Clin Oncol 1998;16:3851-7.

7. Yung WK, Albright RE, Olson J, et al. A phase II study of temozolomide VS. procarbazine in patients with glioblastoma multiforme at first relapse. Br J Cancer 2000;83:588-93.

8. Yung WK, Prados MD, Yaya-Tur R, et al. Multicenter phase II trial of temozolomide in patients with anaplastic astrocytoma or anaplastic oligoastrocytoma at first relapse, Temodal Brain Tumor Group. J Clin Oncol 1999;17:2762-71.

9. Stupp R, Dietrich PY, Ostermann Kraljevic S, et al. Promising survival for patients with newly diagnosed glioblastoma multiforme treated with concomitant radiation plus temozolomide followed by adjuvant temozolomide. J Clin Oncol 2002;20:1375-82.

10. Stupp R, Mason WP, van den Bent MJ, et al. Radiotherapy plus concomitant and adjuvant temozolomide for glioblastoma. N Engl J Med 2005;352:987-96.

11. Westphal M, Hilt DC, Bortey E, et al. A phase III trial of local chemotherapy with biodegradable carmustine (BCNU) wafers (Gliadel wafers) in patients with primary malignant glioma. Neuro Oncol 2003;5:79-88.

12. La Rocca RV, Hodes I, Villanueva WG, et al. A phase II study of radiation with concomitant and then sequential temozolomide(TM2) in patients with newly diagnosed supratentorial high-grade malignant glioma who have undergone surgery with (armustine (SCNU) wafer insertion. Society of Neuro-Oncology, Orlando, Florida, 2006;8(4):445, Abstract 1A-28.

13. Reardon DA, Zalutsky MR, Akabani G, et al. A pilot study: ${ }^{131}$ I-Antitenascin monoclonal antibody 81C6 to deliver a 44-Gy resection cavity boost. Neuro-Oncol 2008;10:182-89.

14. Vredenburgh JJ, Desjardins A, Herndon JE II, et al. Bevacizumab and Irinotecan: an active regimen against recurrent glioblastoma multiforme. J Clin Oncol 2007;25:4722-9.

15. Vredenburgh JJ, Desjardins A, Herndon JE II, et al. Phase II trial of bevacizumab and irinotecan in recurrent malignant glioma. Clin Cancer Res 2007;13:1253-9.

16. Affront Ml, Heery C, Rich J, et al. Overall survival of primary glioblastoma (GBM) patients (pts) receiving Carmustine (8CNU) wafers followed by radiation (RT) and concurrent Temozolomide (TMZ) plus rotational multi-agent chemotherapy, American Society of Clinical Oncology, Chicago, Illinois, 2007.

17. Maxwell JA, Johnson SP, Quinn JA, et al. Quantitative analysis of 06-alkylguanine-DNA alkyltransferase in malignant glioma. Mol Cancer Ther 2006;5:2531-9.

Henry S. Friedman, MD receives clinical research support from Schering-Plough, Genentech, Eisai, Inc. and Bradmer Pharmaceuticals. He is also a paid consultant to Genentech, Eisai, Inc., and Bradmer Pharmaceuticals. 
David A. Reardon, M.D. is a paid consultant to Genentech, Inc., Bradmer Pharmaceuticals, Adnexus Pharmaceuticals and Merck KGaA. 